\title{
Essential spectrum of a periodic elastic waveguide may contain arbitrarily many gaps
}

\section{Taskinen, Jari}

2010

Taskinen , J , Nazarov , S A \& Ruotsalainen , K 2010 , ' Essential spectrum of a periodic elastic waveguide may contain arbitrarily many gaps ' , Applicable Analysis , vol. 89 , no. 1 , pp. 109-124 . https://doi.org/10.1080/00036810903479715

http://hdl.handle.net/10138/24387

https://doi.org/10.1080/00036810903479715

submittedVersion

Downloaded from Helda, University of Helsinki institutional repository.

This is an electronic reprint of the original article.

This reprint may differ from the original in pagination and typographic detail.

Please cite the original version. 


\title{
ESSENTIAL SPECTRUM OF A PERIODIC ELASTIC WAVEGUIDE MAY CONTAIN ARBITRARILY MANY GAPS
}

\author{
SERGEY A. NAZAROV, KEIJO RUOTSALAINEN, AND JARI TASKINEN
}

\begin{abstract}
We construct a family of periodic elastic waveguides $\Pi_{h}$, depending on a small geometrical parameter, with the following property: as $h \rightarrow+0$, the number of gaps in the essential spectrum of the elasticity system on $\Pi_{h}$ grows unboundedly.
\end{abstract}

\section{INTRODUCTION AND FORMULATION OF THE PROBLEM.}

We study the spectral problem for the linearized elasticity system with the traction-free boundary conditions. The goal is to show that in the case the elastic body is a suitable infinite waveguide with periodically positioned cells, the essential spectrum contains gaps, for the number of which we are able to prove a lower bound. Moreover, we construct examples where the lower bound can be made larger than any given number.

For scalar equations and Maxwell's system in infinite periodic media there are many known examples with spectral gaps (see [18, 12, 13, 8, 7, 31] and others). To open a gap, one usually considers differential operators with piecewise constant contracting coefficients and tunes the parameters. Results on gaps in essential spectra of waveguides, infinite in one direction, are much fewer; we mention the papers $[29,9,6,3]$, where a gap is detected using asymptotic analysis of the Helmholtz equation in a singularly perturbed cylindrical waveguide. An approach based on parameter-dependent Korn-type inequalities [25] was proposed in [24, 26, 28]. It permits to detect a gap for periodic homogeneous elastic waveguide of a specific shape with partly clamped surface (cf. fig. 1.1, a)). This approach was modified in $[27,5]$ to cover waveguides with traction-free surfaces of various shapes and elastic properties, in particular, the one in fig. 1.1, b). However, these methods are able to ensure the detection of only one gap. We develop in this paper a new approach which enables to open as many gaps as we wish when a geometric parameter tends to 0 , see our main results Theorem 6.1 and Corollary 6.2.

We consider the elasticity problem in a domain $\Pi_{h}$ which is a periodic waveguide depending on a small geometric parameter $h>0$. The parameter describes the width of ligaments connecting massive periodicity cells, and thus taking the limit $h \rightarrow 0^{+}$, the resulting domain is a disjoint union of countably many bounded domains

1991 Mathematics Subject Classification. primary 35J20, secondary 35P99.

Key words and phrases. linear elasticity system; periodic waveguide; spectral gap.

The first named author was partially supported by the Academy of Finland grant no. 127245 and by RFFI, grant 09-01-00759. The third named author was partially supported by the Väisälä Foundation of the Finnish Academy of Science and Letters. 


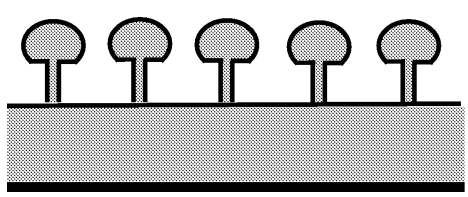

a)

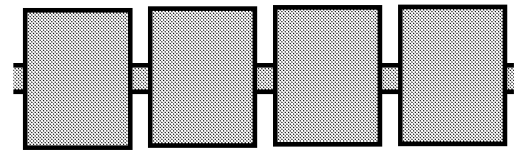

b)

Figure 1.1. Periodic waveguides.

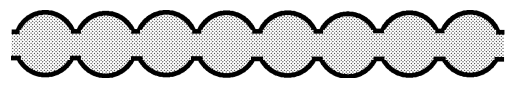

a)

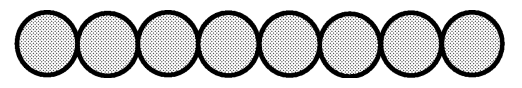

b)

Figure 1.2. The effect of $h \rightarrow 0$.

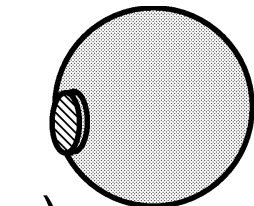

a) b)

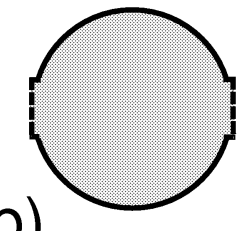

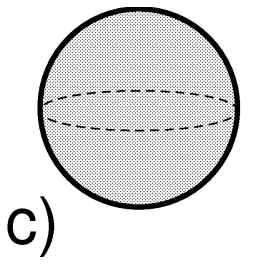

FiguRE 1.3. The cells, real three-dimensional and two-dimensional dummy, and limiting one $(h=0)$.

which are translates of a (bounded) cell $\varpi$ (see fig. 1.2). The spectral structure of the problem on $\Pi_{h}$ is in some sense a perturbation of the discrete spectrum of the elasticity problem on $\varpi$.

Let us proceed with the details. By $\varpi$ we denote a bounded domain in $\mathbb{R}^{3}$

$$
\varpi \subset\left\{x=(y, z): y=\left(y_{1}, y_{2}\right) \in \mathbb{R}^{2},|z|<1 / 2\right\}
$$

having Lipschitz boundary $\partial \varpi$. We assume that the points $\mathcal{O}^{ \pm}=(0,0, \pm 1 / 2)$ are contained in the boundary and that, in some neighbourhood of them, $\partial \varpi$ is of smoothness $C^{3}$.

Let $\Omega_{h}$ denote the thin infinite straight "needle" $\Omega_{h}=\omega_{h} \times \mathbb{R}$, where $\omega \subset \mathbb{R}^{2}$ is a domain such that $\bar{\omega}$ is compact and $\partial \omega$ is smooth, and

$$
\omega_{h}:=h \omega=\left\{y \in \mathbb{R}^{2}: h^{-1} y \in \omega\right\} .
$$

We consider the periodic waveguide $\Pi_{h}$ (fig. 1.2, a))

$$
\Pi_{h}=\Omega_{h} \cup \bigcup_{j \in \mathbb{Z}} \varpi(j)
$$


consisting of the needle $\Omega_{h}$ and the periodic family of bodies

$$
\varpi(j)=\{x:(y, z-j) \in \varpi\}, j \in \mathbb{Z}:=\{0, \pm 1, \pm 2, \ldots\} .
$$

(One could for example imagine the sets $\varpi(j)$ as "beads" in an infinite "necklace" $\left.\Pi_{h}.\right)$ The set

$$
\varpi_{h}=\left\{x \in \Pi_{h}:|z|<1 / 2\right\}=\varpi \cup\left(\omega_{h} \times(-1 / 2,1 / 2)\right)
$$

is called a periodicity cell of the quasi-cylinder $\Pi_{h}$ (see fig. 1.3, a) and b)). At $h=0$ the set (1.3) turns into a union of disconnected domains (1.4) (fig. 1.2 and fig. 1.3, c)). Let $h \in\left(0, h_{0}\right]$, where $h_{0}>0$ is fixed, no matter how. For every such $h$ we consider the spectral elasticity problem expressed in matrix form ([20], [22])

$$
\begin{aligned}
D\left(-\nabla_{x}\right)^{\top} A D\left(\nabla_{x}\right) u^{h} & =\varrho \Lambda^{h} u^{h} \text { in } \Pi_{h}, \\
D(\nu(x))^{\top} A D\left(\nabla_{x}\right) u^{h} & =0 \text { almost everywhere on } \partial \Pi_{h} .
\end{aligned}
$$

Here $\varrho>0$ is the material density which is assumed constant for simplicity, $\Lambda^{h}=\tau_{h}^{2}$ is a spectral parameter and $\tau_{h}$ is the oscillation frequency. Furthermore, $\nabla_{x}$ is the gradient, $\Delta_{x}=\nabla_{x} \cdot \nabla_{x}, u^{h}=\left(u_{1}^{h}, u_{2}^{h}, u_{3}^{h}\right)$ stands for the displacement vector, $\nu=\left(\nu_{1}, \nu_{2}, \nu_{3}\right)$ is the unit vector of the outward normal defined for almost all points of the Lipschitz surface $\partial \Pi_{h}$. The matrix $A$ consists of the elastic moduli, and it is assumed constant, symmetric and positive definite, i.e. the elastic material of the waveguide is, for simplicity, homogeneous. (All considerations can easily be adapted to heterogeneous elastic materials with smooth, periodic $A$ and $\varrho$.$) Moreover, D\left(\nabla_{x}\right)$ is the $6 \times 3$-matrix of first order differential operators,

$$
D(x)=\left(\begin{array}{cccccc}
x_{1} & 0 & 2^{-1 / 2} x_{2} & 2^{-1 / 2} & 0 & 0 \\
0 & x_{2} & 2^{-1 / 2} x_{1} & 0 & 2^{-1 / 2} x_{3} & 0 \\
0 & 0 & 0 & 2^{-1 / 2} x_{1} & 2^{-1 / 2} x_{2}
\end{array}\right)^{\top}
$$

Hence,

$$
D\left(\nabla_{x}\right) u=\left(\varepsilon_{11}(u), \varepsilon_{22}(u), \sqrt{2} \varepsilon_{21}(u), \sqrt{2} \varepsilon_{13}(u), \sqrt{2} \varepsilon_{32}(u), \varepsilon_{33}(u)\right)^{\top},
$$

since the Cartesian components of the strain tensor are given by the Cauchy formulas

$$
\varepsilon_{j k}\left(u^{h}\right)=\frac{1}{2}\left(\frac{\partial u_{j}^{h}}{\partial x_{k}}+\frac{\partial u_{k}^{h}}{\partial x_{j}}\right) .
$$

Finally, the stress column has a structure similar to (1.8), and is defined by the Hooke law as

$$
\sigma(u)=A \varepsilon(u),
$$

hence, the boundary condition (1.7) has the interpretation that the surface $\partial \Pi_{h}$ is free of traction.

The spectral problem (1.6)-(1.7) admits the variational formulation, [19], [21].

$$
\left(A D\left(\nabla_{x}\right) u, D\left(\nabla_{x}\right) v\right)_{\Pi_{h}}=\Lambda_{h}(\varrho u, v)_{\Pi_{h}}, \quad v \in H^{1}\left(\Pi_{h}\right)^{3},
$$

where $(u, v)_{\Pi_{h}}=\sum_{i=1}^{3} \int_{\Pi_{h}} u_{i} v_{i} d x$ is the natural scalar product in the Lebesgue space $L^{2}\left(\Pi_{h}\right)^{3}$. (The superindex 3 indicates here that the function space consists of $\mathbb{R}^{3}$ valued functions. Later on, it will be necessary to consider also complex-valued 
spaces, but the notation is still the same. The superindex will neither be displayed in the notation of the norm of a function space, nor in a scalar product.) The bilinear form (1.9) is closed and positive Hermitian in the Sobolev space $H^{1}\left(\Pi_{h}\right)^{3}$. The Birman-Krein-Vishik theory (see [4, Ch.10] and cf. [2]) can therefore be applied to transform (1.7) to the abstract formulation

$$
\mathcal{T}^{h} u^{h}=\varrho \Lambda^{h} u^{h}
$$

where $\mathcal{T}^{h}$ is an unbounded self-adjoint positive operator in $L^{2}\left(\Pi_{h}\right)^{3}$ and consequently, its spectrum $\Sigma(\mathcal{T})$ lies in $\overline{\mathbb{R}}_{+}=[0, \infty)$. Since the embedding $H^{1}\left(\Pi_{h}\right) \subset$ $L^{2}\left(\Pi_{h}\right)$ is not compact in the unbounded domain $\Pi_{h}$, the essential spectrum $\Sigma_{\text {ess }}(\mathcal{T})$ is not empty (cf. [4, Th.10.1.5]).

\section{BAND-GAP STRUCTURF OF THF SPFCTRIM.}

We recall the definition of the Gelfand transform (see [10] and, e.g. [16, 30, 17] for more details):

$$
v(y, z) \mapsto V(y, z ; \eta)=\frac{1}{\sqrt{2 \pi}} \sum_{m \in \mathbb{Z}} \exp (-i \eta(z+m)) v(y, z+m),
$$

where $(y, z) \in \Pi_{h}$ on the left, $\eta \in[0,2 \pi)$, and $(y, z) \in \varpi_{h}$ on the right. This operator is an isomorphism from $L^{2}\left(\Pi_{h}\right)^{3}$ onto $L^{2}\left(0,2 \pi ; L^{2}\left(\varpi_{h}\right)^{3}\right)$ and from $H^{1}\left(\Pi_{h}\right)^{3}$ onto $H^{1}\left(0,2 \pi ; H_{\text {per }}^{1}\left(\varpi_{h}\right)^{3}\right)$ (see e.g. [30, $\left.\S 3.4\right]$ and [23, Cor. 3.4.3] ). Here $L^{2}\left(0,2 \pi ; L^{2}\left(\varpi_{h}\right)^{3}\right)$ consists of $L^{2}\left(\varpi_{h}\right)^{3}$-valued (complex) $L^{2}$-functions on $[0,2 \pi]$, the space $H^{1}\left(0,2 \pi ; H_{\text {per }}^{1}\left(\varpi_{h}\right)^{3}\right)$ is defined analogously, and $H_{\text {per }}^{1}\left(\varpi_{h}\right)^{3}$ is the space of $\mathbb{C}^{3}$-valued Sobolev-functions 1 -periodic with respect to $z$.

Using the Gelfand transform, the problem (1.6) turns into the following model spectral problem in the periodicity cell (1.5):

$$
\begin{aligned}
& {\overline{D\left(-\nabla_{y},-\partial_{z}-i \eta\right)}}^{\top} A D\left(\nabla_{y}, \partial_{z}+i \eta\right) V^{h}=\varrho M^{h} V^{h} \quad \text { in } \varpi_{h}, \\
& \overline{D(\nu(x))}^{\top} A D\left(\nabla_{y}, \partial_{z}+i \eta\right) V^{h}=0 \quad \text { in } v_{h}=\partial \varpi_{h} \backslash\left(\overline{\omega_{h}^{+} \cup \omega_{h}^{-}}\right) \text {, } \\
& V^{h}(y, 1 / 2)=V^{h}(y,-1 / 2), \partial_{z} V^{h}(y,+1 / 2)=\partial_{z} V^{h}(y,-1 / 2) \text { for } y \in \omega_{h},
\end{aligned}
$$

where $V^{h}$ and $M^{h}$ also depend on dual variable $\eta \in[0,2 \pi]$. Notice that the periodicity conditions are imposed only on the small cross-sections $\omega^{ \pm}=\omega_{h} \times\{ \pm 1 / 2\}$ of the needle.

The weak formulation of the problem $(2.2)$ is to find $V^{h} \in H_{\text {per }}^{1}\left(\varpi_{h}\right)^{3}, V \neq 0$, and $M^{h}$ such that

$$
\left(A D\left(\nabla_{y}, \partial_{z}\right)\left(e^{i \eta z} V^{h}\right), D\left(\nabla_{y}, \partial_{z}\right)\left(e^{i \eta z} U^{h}\right)\right)_{\varpi_{h}}=\varrho M^{h}\left(e^{i \eta z} V^{h}, e^{i \eta z} U^{h}\right)_{\varpi_{h}},
$$

for all $U \in H_{\text {per }}^{1}\left(\varpi_{h}\right)^{3}$; the inner product is that of $L^{2}\left(\varpi_{h}\right)^{3}$. For every real $\eta$, the sesquilinear form on the left hand side of (2.3) is Hermitian and positive on $H_{\text {per }}^{1}\left(\varpi_{h}\right)^{3}$, and it is denoted by

$$
a_{\eta}\left(U, V, \varpi_{h}\right) .
$$

Again by the Birman-Krein-Vishik theory, the problem (2.2) is associated with a self-adjoint semi-bounded operator $T(\eta)$ in $L^{2}\left(\varpi_{h}\right)^{3}$ (see [4, Ch.10]). Since the 
domain $\varpi_{h}$ is bounded, the embedding $H_{\text {per }}^{1}\left(\varpi_{h}\right)^{3} \subset L^{2}\left(\varpi_{h}\right)^{3}$ is compact, and the spectrum of the problem is discrete. The numbers

$$
0 \leq M_{1}^{h}(\eta) \leq M_{2}^{h}(\eta) \leq \ldots \leq M_{p}^{h}(\eta) \leq \ldots \rightarrow+\infty
$$

constitute the eigenvalue sequence (counting multiplicities) for the model problem, and the corresponding eigenvectors are denoted by $V_{(p)}^{h} \in H_{\text {per }}^{1}\left(\varpi_{h}\right)^{3}$ (the dependence of $\eta$ is not displayed). We require the orthogonality and normalization conditions

$$
\left(V_{(p)}^{h}, V_{(q)}^{h}\right)_{\varpi_{h}}=\delta_{p, q}, p, q=1,2, \ldots
$$

For the following fact, see [30, Th. 3.4.6], [23, Th. 2.1], and cf. [16], [17].

Theorem 2.1. A number $\Lambda_{h}$ belongs to the resolvent set or the discrete spectrum of $\mathcal{T}^{h}$, if and only if it does not coincide with a $M_{p}^{h}(\eta)$ for any $\eta \in[0,2 \pi]$ and $p$.

Hence, the spectrum gets the band-gap structure, namely

$$
\Sigma\left(\mathcal{T}^{h}\right)=\Sigma_{\mathrm{ess}}\left(\mathcal{T}^{h}\right)=\bigcup_{p=1}^{\infty} \Upsilon_{p}^{h}
$$

where

$$
\Upsilon_{p}^{h}=\left\{M_{p}^{h}(\eta): \eta \in[0,2 \pi)\right\} .
$$

It is known that the functions $\mathbb{R} \ni \eta \mapsto M_{p}^{h}(\eta)$ are continuous (see [14, Ch.7]) and $2 \pi$-periodic (replacing $\eta$ by $\eta+2 \pi k$, the function $e^{-i 2 \pi k z} V_{(p)}^{h}$ is again an eigenfunction with eigenvalue $M_{p}^{h}(\eta)$, for all $k \in \mathbb{Z}$ ). Hence, (2.8) is a closed segment. However, the authors do not know, if it is possible that

$$
M_{q}^{h}(\eta)=M_{q_{0}}^{h}=\text { const. for } \eta \in\left[\eta_{0}, \eta_{1}\right) \subset[0,2 \pi), \eta_{1}>\eta_{0} .
$$

Under the condition (2.5), the operator $\mathcal{T}^{h}$ in (1.10) gets the eigenvalue $M_{q_{0}}^{h}$ of infinite multiplicity. If (2.9) does not occur for any $q$, the spectrum $\Sigma\left(\mathcal{T}^{h}\right)$ is fully continuous. It follows from $[11,1.5 .1]$ that, if $\eta_{0}<\eta_{1}$ in $(2.9)$, then $\eta_{0}=0$ and $\eta_{1}=2 \pi$.

On the other hand the spectral structure (2.7) does not automatically provide gaps because the bands (2.8) may cover the entire ray $[0,+\infty)$. The idea in the following is to consider the elasticity problem on the bounded domain which is obtained as the limit of the periodicity cell $\varpi_{h}$ as $h \rightarrow 0^{+}$(fig. 1.3, c)); this has a discrete spectrum consisting of eigenvalues (3.3). We shall show that for a given finite number of indices $p$, the band $\Upsilon_{p}^{h}$ can be made to stay within an arbitrarily small distance of the eigenvalue $\mu_{p}$ for small enough $h$. As a consequence, existence of gaps (as many as desired) will be proven.

\section{Spectrum of the limit model problem.}

We next consider a limit model problem on the isolated elastic body $\varpi$. Consider the problem $(2.2)$ at $h=0$ (fig.1.2, b)); then the ligaments of $\Pi_{h}$ vanish, and 
the periodicity conditions of (2.2) cannot be stated any more, and the problem is formulated for the unknown function $V$ on $\varpi$ as

$$
\begin{array}{ll}
{\overline{D\left(-\nabla_{y},-\partial_{z}-i \eta\right)}}^{\top} A D\left(\nabla_{y}, \partial_{z}+i \eta\right) V=\varrho M V & \text { in } \varpi, \\
\overline{D(\nu(x))}{ }^{\top} A D\left(\nabla_{y}, \partial_{z}+i \eta\right) V=0 & \text { in } \partial \varpi .
\end{array}
$$

However, for any $\eta$ this problem has the same eigenvalues as the case $\eta=0$ : if $v$ is a solution to the problem (3.2), below, with the eigenvalue $M$, then $V=\exp (-i \eta z) v$ solves (3.1) with the same eigenvalue $M$. Thus, the case $\eta \neq 0$ has no additional interest here, and we are led to consider the standard spectral problem for the isolated elastic body $\varpi$ corresponding to the case $\eta=0$ (see fig. 1.3, c)),

$$
\begin{array}{lr}
{\overline{D\left(-\nabla_{y},-\partial_{z}\right)}}^{\top} A D\left(\nabla_{y}, \partial_{z}\right) v=\varrho M v & \text { in } \varpi, \\
\overline{D(\nu(x))}^{\top} A D\left(\nabla_{y}, \partial_{z}\right) v=0 & \text { in } \partial \varpi .
\end{array}
$$

Let $a(u, v, \varpi)$ be the form corresponding to the problem (3.2), defined as in (2.3)(2.4) for $\eta=0$. It is Hermitian, positive, sesquilinear and closed on $H^{1}(\varpi)^{3}$. We denote the eigenvalue sequence (arranged as an increasing sequence counting multiplicities) by

$$
0=\mu_{1}=\ldots=\mu_{6}<\mu_{7} \leq \mu_{8} \leq \ldots \leq \mu_{p} \leq \ldots \rightarrow+\infty .
$$

Here $\mu_{1} \ldots \mu_{6}$ are the eigenvalues of the rigid motions $x \mapsto a+b \times x$, which include translations (the constant vector $b \in \mathbb{R}^{3}$ is zero) and rotations $\left(\mathbb{R}^{3} \ni a=0\right)$. For every $p$, let $v_{(p)}$ be the eigenfunction corresponding to $\mu_{p}$, normalized and orthogonal in $L^{2}(\varpi)^{3}$ :

$$
\left(v_{(p)}, v_{(q)}\right)_{\varpi}=\delta_{p, q}, p, q=1,2, \ldots
$$

We recall that by the $\max -\min$ principle (see, e.g., [4, Th.10.2.2])

$$
\mu_{j}=\max _{\mathcal{H}_{j}} \inf _{\mathcal{U} \in \mathcal{H}_{j} \backslash\{0\}} \frac{a(\mathcal{U}, \mathcal{U} ; \varpi)}{\left\|\mathcal{U} ; L^{2}(\varpi)\right\|^{2}},
$$

where $\mathcal{H}_{j}$ stands for any subspace in $H^{1}(\varpi)^{3}$ of codimension $j-1$, in particular, $\mathcal{H}_{1}=H^{1}(\varpi)^{3}$.

At the end of this section we derive pointwise estimates for the eigenfunctions $v_{(p)}$. Let us denote by $B(a, r)$ the open ball with center $a \in \mathbb{R}^{3}$ and radius $r>0$. We recall that the boundary $\partial \varpi$ was by definition $C^{3}$ in a neighbourhood of $\mathcal{O}^{ \pm}$. Hence, denoting $\varpi_{ \pm}^{j}:=\varpi \cap B\left(\mathcal{O}^{ \pm}, r_{j}\right), j=1,2,3$, and assuming the numbers $r_{3}>r_{2}>r_{1}>0$ small enough, the boundaries of $\partial \varpi_{ \pm}^{j}$ are sufficiently smooth so that we can apply the local elliptic regularity estimates for systems (see [1]). As a consequence, the following lemma can be proven:

Lemma 3.1. The pointwise estimates

$$
\begin{aligned}
& \left|v_{(p)}(x)\right| \leq c_{\varpi}\left(1+\mu_{p}\right), \\
& \left|\nabla_{x} v_{(p)}(x)\right| \leq c_{\varpi}\left(1+\mu_{p}\right)^{3 / 2}
\end{aligned}
$$

hold for all $x \in \varpi_{+}^{1}$. 


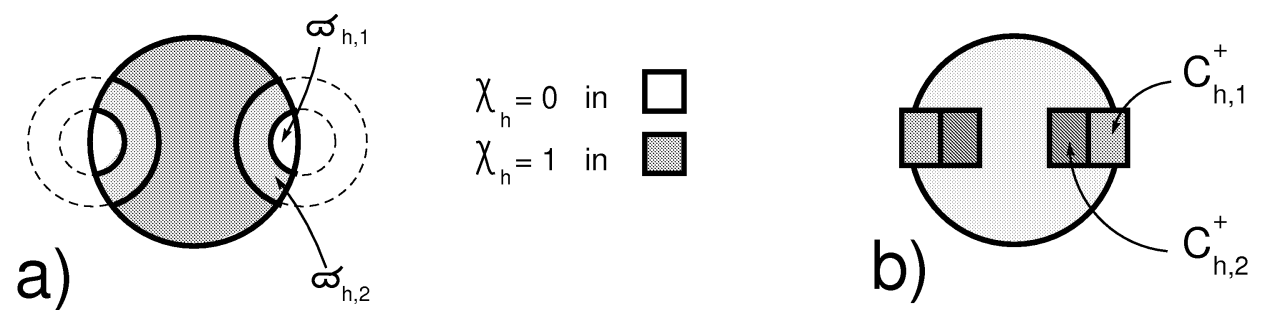

Figure 4.1. The cut-off and small cylinders.

Proof. We apply the local estimates $\left[1\right.$, Ch. 10] to the problem (3.2) with $v:=v_{(p)}$ and $f:=\mu_{p} v_{(p)}$. We thus find for every $l=0,1,2, \ldots$ a constant $C^{\gamma}=C_{\varpi, l, j}>0$ such that

$$
\left\|v_{(p)} ; H^{l+1}\left(\varpi_{ \pm}^{j}\right)\right\| \leq C\left(\mu_{p}\left\|v_{(p)} ; H^{l-1}\left(\varpi_{ \pm}^{j+1}\right)\right\|+\left\|v_{(p)} ; L^{2}\left(\varpi_{ \pm}^{j+1}\right)\right\|\right)
$$

for $j=1,2$. Moreover, $\left\|v_{(p)} ; L^{2}\left(\varpi_{ \pm}^{j+1}\right)\right\| \leq 1$, by the normalization (3.4). The Korn inequality on $\varpi_{ \pm}$(see, e.g., [15]) also implies

$$
\left\|v_{(p)} ; H^{1}\left(\varpi_{ \pm}^{j}\right)\right\|^{2} \leq\left\|v_{(p)} ; H^{1}(\varpi)\right\|^{2}
$$

$(3.8) \leq C\left(a\left(v_{(p)}, v_{(p)} ; \varpi\right)+\left\|v_{(p)} ; L^{2}(\varpi)\right\|^{2}\right)=C\left(\mu_{p}+1\right)\left(v_{(p)}, v_{(p)}\right)_{\varpi}=C\left(\mu_{p}+1\right)$.

Taking $l=1, j=2$ in (3.7) we thus obtain

$$
\left\|v_{(p)} ; H^{2}\left(\varpi_{ \pm}^{2}\right)\right\| \leq C_{l}\left(\mu_{p}\left\|v_{(p)} ; L^{2}\left(\varpi_{ \pm}^{3}\right)\right\|+\left\|v_{(p)} ; L^{2}\left(\varpi_{ \pm}^{3}\right)\right\|\right) \leq C_{1}\left(\mu_{p}+1\right)
$$

and for $l=2, j=1$,

$$
\begin{aligned}
& \left\|v_{(p)} ; H^{3}\left(\varpi_{ \pm}^{1}\right)\right\| \leq C_{l}\left(\mu_{p}\left\|v_{(p)} ; H^{1}\left(\varpi_{ \pm}^{2}\right)\right\|+\left\|v_{(p)} ; L^{2}\left(\varpi_{ \pm}^{2}\right)\right\|\right) \\
\leq & C_{2}\left(\mu_{p}+1\right)^{3 / 2} .
\end{aligned}
$$

In view of the standard embeddings $H^{2}\left(\varpi_{+}^{2}\right) \subset C_{B}\left(\varpi_{+}^{1}\right)$ and $H^{3}\left(\varpi_{+}^{1}\right) \subset C_{B}^{1}\left(\varpi_{+}^{1}\right)$, the estimates (3.6) follow from (3.9) and (3.10).

\section{UPPER ESTIMATE FOR THE BANDS.}

In the next two sections we consider the relation of the spectral band $\Upsilon_{p}^{h}$ with the eigenvalue $\mu_{p}$. We start with an upper estimate for $M_{p}^{h}(\eta) \in \Upsilon_{p}^{h}$ in terms of $\mu_{p}$.

Lemma 4.1. There exists a decreasing sequence $0<h_{j}<h_{j-1}$ and a constant $C>0$, depending only on $\varpi, A$, and $\varrho$, such that

$$
M_{j}^{h}(\eta) \leq \mu_{j}+C h j\left(1+\mu_{j}\right)^{2}
$$

for all $j \in \mathbb{N}, h \leq h_{j}$ and $\eta \in[0,2 \pi)$.

Proof. Let us fix a constant $c_{\chi}>0$ such that $3 c_{\chi}<r_{1}$ (see above Lemma 3.1 for this number). Given $h$, we denote by $\chi_{h}: \varpi_{h} \rightarrow[0,1]$ a smooth cut-off function which is equal to 0 on the subset

$$
\varpi_{h, k}:=\varpi_{h} \cap\left(B\left(\mathcal{O}^{+}, k c_{\chi} h\right) \cup B\left(\mathcal{O}^{-}, k c_{\chi} h\right)\right),
$$


if $k=1$, and equal to 1 on $\varpi_{h} \backslash \varpi_{h, 2}$, see fig. 4.1, a). We can also require that for some constant $C_{\varpi}>0$

$$
\left|\nabla_{x} \chi_{h}(x)\right| \leq C_{\varpi} h^{-1}
$$

For all $j \in \mathbb{N}$, we set $\mathcal{V}_{(j)}^{h}(x ; \eta)=\chi_{h}(x) v_{(j)}(x ; \eta)$ for $x \in \varpi$, and extend the product as 0 for $x \in \varpi_{h} \backslash \varpi$. The choice of $c_{\chi}$ guarantees that the extensions become smooth and that the following holds:

$$
\operatorname{supp} \mathcal{V}_{(j)}^{h} \subset \varpi \text { and } \operatorname{supp}\left(\mathcal{V}_{(j)}^{h}-v_{(j)}\right) \subset \varpi_{ \pm}^{1} ;
$$

the latter can be achieved for small enough $h$ which we from now on assume.

Using the notation of (4.2) with $k=2$, and (3.6), we can thus bound

$$
\left\|v_{(j)}-\mathcal{V}_{(j)}^{h} ; L^{2}(\varpi)\right\|^{2} \leq \int_{\varpi, 2 \cap\rceil} c_{\varpi}\left(1+\mu_{j}\right)^{2} d x \leq C_{\varpi} h^{3}\left(1+\mu_{j}\right)^{2},
$$

since the volume of the set $\varpi_{h, 2}$ is of order $h^{3}$. The following $L^{1}$-estimates can be proven in the same way:

$$
\begin{aligned}
& \left\|v_{(j)}-\mathcal{V}_{(j)}^{h} ; L^{1}(\varpi)\right\| \leq C_{\varpi} h^{3}\left(1+\mu_{j}\right), \\
& \left\|\nabla_{x} v_{(j)}-\nabla_{x} \mathcal{V}_{(j)}^{h} ; L^{1}(\varpi)\right\| \leq \int_{\varpi, 2 \cap \varpi}\left(\left|\nabla_{x} v_{(j)}\right|+\left|\nabla_{x} \chi_{h}\right|\left|v_{(j)}\right|\right) d x \\
\leq & C_{\varpi}\left(h^{3}\left(1+\mu_{j}\right)^{3 / 2}+h^{2}\left(1+\mu_{j}\right)\right) .
\end{aligned}
$$

Notice that also by (3.6), (4.3), (4.4),

$$
\begin{aligned}
& \left\|v_{(j)} ; L^{\infty}\left(\varpi_{ \pm}^{1}\right)\right\| \leq C_{\varpi}\left(1+\mu_{j}\right), \\
& \left\|\nabla_{x} v_{(j)} ; L^{\infty}\left(\varpi_{ \pm}^{1}\right)\right\| \leq C_{\varpi}\left(1+\mu_{j}\right)^{3 / 2}, \\
& \left\|\mathcal{V}_{(j)}^{h}-v_{(j)} ; L^{\infty}(\varpi)\right\| \leq C_{\varpi}\left(1+\mu_{j}\right), \\
& \left\|\nabla_{x} \mathcal{V}_{(j)}^{h}-\nabla_{x} v_{(j)} ; L^{\infty}(\varpi)\right\| \leq \sup _{\varpi}\left(\left|\nabla_{x} v_{(j)}\right|+\left|\nabla_{x} \chi_{h}\right|\left|v_{(j)}\right|\right) \\
& \leq C_{\varpi}\left(\left(1+\mu_{j}\right)^{3 / 2}+h^{-1}\left(1+\mu_{j}\right)\right) .
\end{aligned}
$$

For every $j \in \mathbb{N}$ we now pick an $h_{j}, 0<h_{j}<1$, such that $h_{j}<h_{j-1}$ and

$$
\left(1+C_{\varpi}\right) h_{j}\left(1+\mu_{j}\right) \leq 2^{-j-3} ;
$$

and also such that the requirement after (4.4) is met for this $h_{j}$.

Let us fix the index $j$ for the rest of the proof, and consider numbers $h$ satisfying $h<h_{j}$. First note that the choice of the numbers $h_{j}$ and (4.5), (4.9) imply, for all $q \leq j$ and $h \leq h_{j}$.

$$
\begin{aligned}
& \left\|\mathcal{V}_{(q)}^{h}-v_{(q)} ; L^{2}(\varpi)\right\| \leq 2^{-q-3} \quad \text { and } \\
& \left\|\mathcal{V}_{(q)}^{h} ; L^{2}(\varpi)\right\| \leq\left\|v_{(q)} ; L^{2}(\varpi)\right\|+\left\|\mathcal{V}_{(q)}^{h}-v_{(q)} ; L^{2}(\varpi)\right\| \leq 1+2^{-q-3} .
\end{aligned}
$$

We claim that the functions $\mathcal{V}_{(1)}^{h}, \ldots, \mathcal{V}_{(j)}^{h}$ are linearly independent in $L^{2}(\varpi)^{3}$ (hence also in the space $\left.L^{2}\left(\varpi_{h}\right)^{3}\right)$. If not, then there exists an index $p, 1 \leq p \leq j$ and 
numbers $b_{q}$ such that

$$
\mathcal{V}_{(p)}^{h}=\sum_{\substack{q=1 \\ q \neq p}}^{j} b_{q} \mathcal{V}_{(q)}^{h} .
$$

Here we may assume that $0 \leq\left|b_{q}\right| \leq 1$, since if this is not the case from the beginning, we pick up the $q$ with largest $\left|b_{q}\right|$, and change $p$ for this $q$.

From (3.4) and (4.10) we get for every $q \leq j$

$$
\left\|\mathcal{V}_{(q)}^{h} ; L^{2}(\varpi)\right\| \geq\left\|v_{(q)}^{h} ; L^{2}(\varpi)\right\|-\left\|\mathcal{V}_{(q)}^{h}-v_{(q)} ; L^{2}(\varpi)\right\| \geq 1-2^{-q-3}
$$

and, for $q \neq p$, using the Cauchy-Schwartz inequality, and (4.10), (3.4),

$$
\left|\left(v_{(p)}, \mathcal{V}_{(q)}^{h}\right)_{\varpi}\right| \leq\left|\left(v_{(p)}, \mathcal{V}_{(q)}^{h}-v_{(q)}\right)_{\varpi}\right|+\left|\left(v_{(p)}, v_{(q)}\right)_{\varpi}\right| \leq 2^{-q-3} .
$$

Taking the $L^{2}(\varpi)$-inner product with $\mathcal{V}_{(p)}^{h}$ on each side of (4.11), the left hand side is at least $\left(1-2^{-p-3}\right)^{2} \geq 1 / 2$ by (4.12), whereas the right hand side is, by (4.13) and (4.10), at most

$$
\begin{aligned}
& \left.\mid\left(\mathcal{V}_{(p)}^{h}, \sum_{\substack{q=1 \\
q \neq p}}^{j} b_{q} \mathcal{V}_{(q)}^{h}\right)_{\varpi}\right)_{\varpi} \mid \\
= & \left.\left|\left(\mathcal{V}_{(p)}^{h}-v_{(p)}, \mathcal{V}_{(p)}^{h}\right)_{\varpi}\right|+\mid\left(v_{(p)}, \sum_{\substack{q=1 \\
q \neq p}}^{j} b_{q} \mathcal{V}_{(q)}^{h}\right)_{\varpi}\right)_{\varpi} \mid \\
\leq & \left\|\mathcal{V}_{(p)}^{h}-v_{(p)} ; L^{2}(\varpi)\right\|\left\|\mathcal{V}_{(p)}^{h} ; L^{2}(\varpi)\right\|+\sum_{\substack{q=1 \\
q \neq p}}^{j} b_{q}\left|\left(v_{(p)}, \mathcal{V}_{(q)}^{h}\right)_{\varpi}\right| \\
\leq & 2^{-p-3}\left(1+2^{-p-3}\right)+\sum_{q=1}^{j} 2^{-q-3} \leq \frac{1}{3},
\end{aligned}
$$

which contradicts with the previous estimate. Hence, (4.11) cannot hold, and the linear independence is proven.

Let now $\left(b_{p}\right)_{p=1}^{j}$ be any sequence of numbers normalized so that $\sum_{p=1}^{j}\left|b_{p}\right|^{2}=1$, and let

$$
\mathcal{W}_{(j)}^{h}=\sum_{p=1}^{j} b_{p} \mathcal{V}_{(p)}^{h} \quad, \quad W_{j}:=\sum_{p=1}^{j} b_{p} v_{(p)}
$$

hence,

$$
\left\|W_{j} ; L^{2}(\varpi)\right\|=1
$$

since the functions $v_{(p)}$ are orthonormal in $L^{2}(\varpi)^{3}$.

We evaluate $a\left(\mathcal{W}_{(j)}^{h}, \mathcal{W}_{(j)}^{h} ; \varpi\right)$. First, by (3.3), (3.4), and (4.16),

$$
\left|a\left(W_{(j)}, W_{(j)} ; \varpi\right)\right| \leq \mu_{j} .
$$


Moreover.

$$
\begin{aligned}
a\left(\mathcal{W}_{(j)}^{h}, \mathcal{W}_{(j)}^{h} ; \varpi\right) & =a\left(W_{(j)}, W_{(j)} ; \varpi\right)+\sum_{p, q=1}^{j} b_{p} \bar{b}_{q}\left(a\left(\mathcal{V}_{(p)}^{h}-v_{(p)}, v_{(q)} ; \varpi\right)\right. \\
& \left.+a\left(v_{(p)}, \mathcal{V}_{(q)}^{h}-v_{(q)} ; \varpi\right)+a\left(\mathcal{V}_{(p)}^{h}-v_{(p)}, \mathcal{V}_{(q)}^{h}-v_{(q)} ; \varpi\right)\right)
\end{aligned}
$$

The expression for $a(\cdot, \cdot, \varpi)$, the Hölder inequality, and (4.4), (4.7), (4.8), (3.3) imply

$$
\begin{aligned}
& \left|a\left(\mathcal{V}_{(p)}^{h}-v_{(p)}, v_{(q)} ; \varpi\right)\right| \\
\leq & C_{a}\left(\left\|\mathcal{V}_{(p)}^{h}-v_{(p)} ; L^{1}\left(\varpi_{ \pm}^{1}\right)\right\|+\left\|\nabla_{x} \mathcal{V}_{(p)}^{h}-\nabla_{x} v_{(p)} ; L^{1}\left(\varpi_{ \pm}^{1}\right)\right\|\right) \\
\times & \left(\left\|v_{(q)} ; L^{\infty}\left(\varpi_{ \pm}^{1}\right)\right\|+\left\|\nabla_{x} v_{(q)} ; L^{\infty}\left(\varpi_{ \pm}^{1}\right)\right\|\right) \\
\leq & C_{\varpi}\left(h^{3}\left(1+\mu_{j}\right)^{3 / 2}+h^{2}\left(1+\mu_{j}\right)\right)\left(\left(1+\mu_{j}\right)^{3 / 2}+h^{-1}\left(1+\mu_{j}\right)\right) .
\end{aligned}
$$

Taking into account (4.9), this can be bounded by

$$
C_{\varpi} h\left(1+\mu_{j}\right)^{2} .
$$

The third and fourth terms on the right hand side of (4.18) have the same bound. From (4.17)-(4.20) and

$$
\sum_{p, q=1}^{j} b_{p} \bar{b}_{q} \leq \sum_{p, q=1}^{j}\left(\left|b_{p}\right|^{2}+\left|b_{q}\right|^{2}\right) \leq 2 j
$$

we thus obtain

$$
\left|a\left(\mathcal{W}_{(j)}^{h}, \mathcal{W}_{(j)}^{h} ; \varpi\right)\right| \leq \mu_{j}+C_{\varpi} h j\left(1+\mu_{j}\right)^{2} .
$$

In the same vain one can estimate using (4.6), (4.8),

$$
\begin{aligned}
& \left\|\mathcal{W}_{(j)}^{h} ; L^{2}(\varpi)\right\|^{2}=\left(\mathcal{W}_{(j)}^{h}, \mathcal{W}_{(j)}^{h}\right)_{\varpi} \\
= & \left(W_{j}, W_{j}\right)_{\varpi}+\sum_{p, q=1}^{j} b_{p} \bar{b}_{q}\left(\left(\mathcal{V}_{(p)}^{h}-v_{(p)}, v_{(q)}\right)_{\varpi}\right. \\
+ & \left.\left(v_{(p)}, \mathcal{V}_{(q)}^{h}-v_{(q)}\right)_{\varpi}+\left(\mathcal{V}_{(p)}^{h}-v_{(p)}, \mathcal{V}_{(q)}^{h}-v_{(q)}\right)_{\varpi}\right) \\
\geq & 1-C_{\varpi} j h^{3}\left(1+\mu_{j}\right)^{2} .
\end{aligned}
$$

To apply these estimates we use the max-min principle [4, Th.10.2.2]

$$
M_{j}^{h}(\eta)=\max _{\mathcal{H}_{j}} \inf _{\mathcal{U} \in \mathcal{H}_{j} \backslash\{0\}} \frac{a_{\eta}\left(\mathcal{U}, \mathcal{U} ; \varpi_{h}\right)}{\left\|\mathcal{U} ; L^{2}\left(\varpi_{h}\right)\right\|^{2}},
$$

where $\mathcal{H}_{j}$ stands for any subspace in $H_{\text {per }}^{1}\left(\varpi_{h}\right)$ of codimension $j-1$. Since the sequence of functions $\left(\mathcal{V}_{(p)}\right)_{p=1}^{j}$ was shown to be linearly independent we can find from any $\mathcal{H}_{j}$ an element $\mathbf{W}_{(j)}^{h}$ of the form

$$
\mathbf{W}_{(j)}^{h}(x, \eta)=e^{-i \eta z} \mathcal{W}_{(j)}^{h}(x),
$$


where $\mathcal{W}_{(j)}^{h}$ is as in (4.15). Moreover, by the definition of the forms $a_{\eta}$ and $a$ (see (2.3)-(2.4) and the things below (3.2)), and by (4.4), we have

$$
a_{\eta}\left(\mathbf{W}_{(j)}^{h}, \mathbf{W}_{(j)}^{h} ; \varpi_{h}\right)=a\left(\mathcal{W}_{(j)}^{h}, \mathcal{W}_{(j)}^{h} ; \varpi\right),\left\|\mathbf{W}_{(j)}^{h}, L^{2}\left(\varpi_{h}\right)\right\|=\left\|\mathcal{W}_{(j)}^{h}, L^{2}(\varpi)\right\| .
$$

These, together with (4.23), (4.21), (4.22), imply

$$
\begin{aligned}
M_{j}^{h}(\eta) & \leq \frac{a_{\eta}\left(\mathbf{W}_{(j)}^{h}, \mathbf{W}_{(j)}^{h} ; \varpi_{h}\right)}{\left\|\mathbf{W}_{(j)}^{h} ; L^{2}\left(\varpi_{h}\right)\right\|^{2}}=\frac{a\left(\mathcal{W}_{(j)}^{h}, \mathcal{W}_{(j)}^{h} ; \varpi\right)}{\left\|\mathcal{W}_{(j)}^{h} ; L^{2}(\varpi)\right\|^{2}} \\
& \leq \frac{\mu_{j}+C_{\varpi} h j\left(1+\mu_{j}\right)^{2}}{1-C_{\varpi} h^{3} j\left(1+\mu_{j}\right)^{2}} \\
& \leq \mu_{j}+C h j\left(1+\mu_{j}\right)^{2} .
\end{aligned}
$$

\section{LOWER ESTIMATE FOR BANDS.}

We prove the lower estimate for the numbers $M_{j}^{h}(\eta)$, see (5.5). We shall need the following inequality of Korn's type, see [25, Ch. 2]:

\section{Lemma 5.1.}

$$
\left\|V ; L^{2}\left(\varpi_{h} \backslash \varpi\right)\right\|^{2} \leq C_{K} h^{2}\left(a\left(V, V ; \varpi_{h}\right)+\left\|V ; L^{2}\left(\varpi_{h}\right)\right\|^{2}\right),
$$

where $C_{K}$ is independent of $h \in(0,1]$ and $V \in H^{1}\left(\varpi_{h}\right)$.

Proof. Owing to the standard Korn inequality in a bounded Lipschitz domain (see, e.g., [15]) we have

$$
\left\|u ; H^{1}(\varpi)\right\|^{2} \leq c_{\varpi}\left(a(u, u ; \Omega)+\left\|u ; L^{2}(\varpi)\right\|^{2}\right) .
$$

By the one-dimensional Hardy inequality

$$
\int_{0}^{\infty}|U(r)|^{2} d r \leq 4 \int_{0}^{\infty} r^{2}\left|\frac{d U}{d r}\right|^{2} d r, \quad U \in C_{c}^{1}[0,1),
$$

there also holds

$$
\left\|\varrho_{+}^{-1} u ; L^{2}(\varpi)\right\|^{2} \leq C\left\|u ; H^{1}(\varpi)\right\|^{2},
$$

where $\varrho_{ \pm}$is the distance to the point $\mathcal{O}^{ \pm}$. Notice that in order to derive (5.4) from (5.3) we need to multiply $u$ with an appropriate cut-off function $\chi_{ \pm}$, to apply (5.3) to the radial variable $\varrho_{ \pm}$, and integrate in the anglular variables while taking the Jacobian $\varrho_{+}^{2}$ into account. We emphasize that the above multiplication forces us to put the Sobolev norm (instead of the Dirichlet seminorm) on the right hand side of (5.4). A completion argument is used to pass from continuously differentiable functions to Sobolev functions in $(5.3),(5.4)$.

For $k=1,2$, let $C_{h k}^{+}=\omega_{h} \times(-1 / 2+(k-1) l h,-1 / 2+k l h)$ be small cylinders, and let $C_{h k}^{-}$have a similar meaning (see fig. 4.1, b)). We fix the size $l>0$ such that $\varpi_{h} \backslash\left(C_{h 1}^{+} \cup C_{h 1}^{-}\right) \subset \varpi$ (see fig.4.1, b)). Then $C_{h 2}^{ \pm} \subset \varpi$ for a small $h>0$ and $\varrho^{ \pm} \leq c_{\varrho} h$ for $x \in C_{h 2}^{ \pm}$so that, by (5.4) and (5.2), we get

$$
\left\|u ; L^{2}\left(C_{h 2}^{ \pm}\right)\right\|^{2} \leq c h^{2}\left\|u ; H^{1}(\varpi)\right\|^{2} \leq c h^{2}\left(a(u, u ; \Omega)+\left\|u ; L^{2}(\varpi)\right\|^{2}\right)
$$


We go over to the streched coordinates $\xi^{ \pm}=\left(h^{-1}\left(x_{1} \mp 1 / 2, x_{2}, x_{3}\right)\right.$ and transform $C_{h k}^{ \pm}$into the cylinders $C_{1 k}^{ \pm}$of unit size. After applying the coordinate compression $\xi^{ \pm} \mapsto x$, the variant of the Korn's inequality

$$
\left\|\mathbf{u} ; L^{2}\left(C_{11}^{ \pm}\right)\right\|^{2} \leq c_{\omega}\left(a\left(\mathbf{u}, \mathbf{u} ; C_{12}^{ \pm} \cup C_{11}^{ \pm}\right)+\left\|\mathbf{u} ; L^{2}\left(C_{12}^{ \pm}\right)\right\|^{2}\right)
$$

turns into the the relation

$$
h^{-2}\left\|u ; L^{2}\left(C_{h 1}^{ \pm}\right)\right\|^{2} \leq c_{\omega}\left(a\left(u, u ; C_{h 2}^{ \pm} \cup C_{h 1}^{ \pm}\right)+h^{-2}\left\|u ; L^{2}\left(C_{h 2}^{ \pm}\right)\right\|^{2}\right),
$$

which completes the proof.

Lemma 5.2. There exist a constant $C>0$, and, for every $j$, a number $\tilde{h}_{j}>0$, such that

$$
M_{j}^{h}(\eta) \geq \mu_{j}-C h \mu_{j}^{2}
$$

for all $0<h<\tilde{h}_{j}$.

Proof. We consider the eigenvectors $V_{(j)}^{h} \in H_{\text {per }}^{1}\left(\varpi_{h}\right)^{3}$, see (2.6). Since $\varpi \subset \varpi_{h}$ for every $h$, we have $V_{(j)}^{h} \in H^{1}(\varpi)^{3}$. By $(4.1)$, if $h \leq h_{j}$, every $M_{j}^{h}(\eta)$ can be bounded by a positive number depending only on $j$. Thus we can choose for every $j$ a small enough $\tilde{h}_{j}>0$ such that $\tilde{h}_{j}<\tilde{h}_{j-1}$ and such that

$$
\left(1+C_{K}\right) h\left(1+M_{j}^{h}(\eta)\right) \leq 2^{-j-3} \text { for } \eta \in[0,2 \pi), h \in\left(0, \tilde{h}_{j}\right] .
$$

We claim that then the eigenvectors $V_{(1)}^{h}, \ldots, V_{(j)}^{h}$ still remain linearly independent in $L^{2}(\varpi)^{3}$. Otherwise there exists a $p, 1 \leq p \leq j$ and numbers $\beta_{q}$ such that

$$
V_{(p)}^{h}=\sum_{\substack{q=1 \\ q \neq p}}^{j} \beta_{q} V_{(q)}^{h} .
$$

Again one may assume that $0 \leq\left|\beta_{q}\right| \leq 1$, by possibly changing $p$ to the $q$ with largest $\left|\beta_{q}\right|$. We have $a_{\eta}\left(V_{(p)}^{h}, V_{(p)}^{h} ; \varpi_{h}\right)=M_{p}^{h}(\eta)$ because of the normalization (2.6). Hence, using the Korn inequality (5.1), and (5.6) and we get for every $p \leq j$

$$
\left\|V_{(p)}^{h} ; L^{2}\left(\varpi_{h} \backslash \varpi\right)\right\| \leq 2^{-p-3} \text {, hence }\left\|V_{(p)}^{h} ; L^{2}(\varpi)\right\| \geq 1-2^{-p-3} .
$$

and, for $q \neq p$,

$$
\begin{aligned}
& \left|\left(V_{(p)}^{h}, V_{(q)}^{h}\right)_{\varpi}\right| \leq\left|\left(V_{(p)}^{h}, V_{(q)}^{h}\right)_{\varpi_{h}}\right|+\left|\left(V_{(p)}^{h}, V_{(q)}^{h}\right)_{\varpi_{h} \backslash \varpi}\right| \\
\leq & \left\|V_{(p)}^{h} ; L^{2}\left(\varpi_{h} \backslash \varpi\right)\right\|\left\|V_{(q)}^{h} ; L^{2}\left(\varpi_{h} \backslash \varpi\right)\right\| \leq 2^{-q-3} .
\end{aligned}
$$

Taking the $L^{2}(\varpi)^{3}$-inner product with $V_{(p)}^{h}$ on each side of $(5.7)$, the second estimate of (5.8) and (5.9) clearly lead to a contradiction.

Fixing $j$ and also $\eta$, and assuming $h \leq \tilde{h}_{j}$, we start to bound $\mu_{j}$. The sequence $\left(e^{i \eta z} V_{(p)}^{h}\right)_{p=1}^{j}$ is still linearly independent in $L^{2}(\varpi)^{3}$, hence, any subspace $\mathcal{H}_{j} \subset L^{2}(\varpi)^{3}$ of codimension $j-1$ contains a linear combination

$$
\sum_{p=1}^{j} b_{p} e^{i \eta z} V_{(p)}^{h}, \text { such that } \sum_{p=1}^{j}\left|b_{p}\right|^{2}=1 .
$$


We denote

$$
\mathcal{U}_{(j)}^{h}=\sum_{p=1}^{j} b_{p} V_{(p)}^{h} ;
$$

obviously $\left\|\mathcal{U}_{(j)}^{h} ; L^{2}\left(\varpi_{h}\right)\right\|=1$ because of (2.6) and (5.10).

Since $V_{(p)}^{h}$ are the eigenvectors of the operator $T(\eta)$ (see above (2.5)) and (2.5) holds, we have, also by $(2.6),(5.11)$, and $a_{\eta}\left(V_{(p)}^{h}, V_{(p)}^{h} ; \varpi_{h}\right)=M_{p}^{h}(\eta)$, that

$$
\left|a_{\eta}\left(\mathcal{U}_{(j)}^{h}, \mathcal{U}_{(j)}^{h} ; \varpi_{h}\right)\right| \leq M_{j}^{h}(\eta) .
$$

Notice that on the other hand

$$
\left|a_{0}\left(e^{i \eta z} \mathcal{U}_{(j)}^{h}, e^{i \eta z} \mathcal{U}_{(j)}^{h} ; \varpi_{h}\right)\right|=\left|a_{\eta}\left(\mathcal{U}_{(j)}^{h}, \mathcal{U}_{(j)}^{h} ; \varpi_{h}\right)\right| .
$$

By (5.1) and (5.12) we can also estimate

$$
\begin{aligned}
& \left\|e^{i \eta z} \mathcal{U}_{(j)}^{h} ; L^{2}(\varpi)\right\|^{2}=\left\|\mathcal{U}_{(j)}^{h} ; L^{2}(\varpi)\right\|^{2} \\
\geq & \left\|\mathcal{U}_{(j)}^{h} ; L^{2}\left(\varpi_{h}\right)\right\|^{2}-\left\|\mathcal{U}_{(j)}^{h} ; L^{2}\left(\varpi_{h} \backslash \varpi\right)\right\|^{2} \\
\geq & 1-C h^{2}\left(1+a\left(\mathcal{U}_{(j)}^{h}, \mathcal{U}_{(j)}^{h} ; \varpi_{h}\right)\right) \\
\geq & 1-C h^{2}\left(1+M_{j}^{h}(\eta)\right),
\end{aligned}
$$

hence, using (5.12)-(5.14) we obtain

$$
\begin{aligned}
& \frac{a\left(e^{i \eta z} \mathcal{U}_{(j)}^{h}, e^{i \eta z} \mathcal{U}_{(j)}^{h} ; \varpi\right)}{\left\|e^{i \eta z} \mathcal{U}_{(j)}^{h} ; L^{2}(\varpi)\right\|^{2}} \leq \frac{a_{0}\left(e^{i \eta z} \mathcal{U}_{(j)}^{h}, e^{i \eta z} \mathcal{U}_{(j)}^{h} ; \varpi_{h}\right)}{\left\|e^{i \eta z} \mathcal{U}_{(j)}^{h} ; L^{2}(\varpi)\right\|^{2}} \\
= & \frac{a_{\eta}\left(\mathcal{U}_{(j)}^{h}, \mathcal{U}_{(j)}^{h} ; \varpi_{h}\right)}{\left\|\mathcal{U}_{(j)}^{h} ; L^{2}(\varpi)\right\|^{2}} \leq a_{\eta}\left(\mathcal{U}_{(j)}^{h}, \mathcal{U}_{(j)}^{h} ; \varpi_{h}\right)\left(1+C h^{2}\left(1+M_{j}^{h}(\eta)\right)\right) \\
\leq & M_{j}^{h}(\eta)\left(1+C h^{2}\left(1+M_{j}^{h}(\eta)\right)\right) .
\end{aligned}
$$

Again, since $\mathcal{H}_{j}$ was an arbitrary $(j-1)$-codimensional subspace of $L^{2}(\varpi)$, we get from the max-min-principle (3.5)

$$
\begin{aligned}
\mu_{j} & \leq M_{j}^{h}(\eta)\left(1+C h^{2}\left(1+M_{j}^{h}(\eta)\right)\right) \\
& =M_{j}^{h}(\eta)\left(1+C h^{2}\right)+M_{j}^{h}(\eta)^{2} C h^{2} .
\end{aligned}
$$

For the rest of the proof, let us fix the constant $C$ as on the last line of (5.15). Consider the following equation for an unknown $\xi \in \mathbb{R}$ :

$$
\mu_{j}=\xi\left(1+C h^{2}\right)+\xi^{2} C h^{2} .
$$

Its positive solution is

$$
\xi=\frac{1+C h^{2}}{2 C h^{2}}\left(-1+\sqrt{1+\frac{4 C h^{2}}{1+C h^{2}} \mu_{j}}\right) .
$$

Assuming $h$ so small that $4 C h^{2} \mu_{j}<1$ (we redefine $\tilde{h}_{j}$ such that this holds for all $h \leq \tilde{h}_{j}$ ), we obtain from the binomial series $\sqrt{1+\beta}=1+\beta / 2-\beta^{2} / 8+-\ldots \geq$ 
$1+\beta / 2-\beta^{2} / 8$ the following estimate:

$$
\xi \geq \mu_{j}-\mu_{j}^{2} \frac{C h^{2}}{1+C h^{2}} \geq \mu_{j}-\mu_{j}^{2} C h^{2}
$$

Hence, in view of $(5.15),(5.16)$, also $M_{j}^{h}(\eta) \geq \mu_{j}-\mu_{j}^{2} C h^{2}$ holds.

\section{Main Result.}

We are now in a position to formulate our main theorem. Recall that the spectrum (2.7) coincides with the essential spectrum of the original elasticity system (1.6)(1.7), see Theorem 2.1.

Theorem 6.1. Let $j \in \mathbb{N}$ be such that $\mu_{j}>\mu_{j-1}$ in (3.3). Then, for $h \in$ $\left(0, \min \left(h_{j}, \tilde{h}_{j}\right)\right]$, the bands $\Upsilon_{1}^{h}, \ldots, \Upsilon_{j-1}^{h}$ and $\Upsilon_{j}^{h}, \Upsilon_{j+1}^{h}, \ldots$, repectively, are contained in the sets $\left[0, \mu_{j-1}+A h j\left(1+\mu_{j-1}\right)^{2}\right]$ and $\left[\mu_{j}-A h^{2} \mu_{j}^{2},+\infty\right)$, where $A>0$ depends on neither $h$, nor $j$.

Choosing ho small that

$$
A h j\left(1+\mu_{j-1}\right)^{2}+A h^{2} \mu_{j}^{2}<\mu_{j}-\mu_{j-1}
$$

the spectrum (2.7) (and thus also the essential spectrum of the elasticity system (1.6)-(1.7)) get a gap between $\Upsilon_{j-1}^{h}$ and $\Upsilon_{j}^{h}$.

Proof. By (6.1) and Lemmas 4.1 and 5.2, the right end point of $\Upsilon_{j-1}^{h}$ is strictly smaller than the left end point of $\Upsilon_{j}^{h}$. It is thus enough to recall that the left (resp. right) end point of the segment $\Upsilon_{p}^{h}$ is for all $p>j$ (resp. $p<j-1$ ) at least as large (resp. small) as the left (resp. right) end point of $\Upsilon_{j}^{h}\left(\right.$ resp. $\left.\Upsilon_{j-1}^{h}\right)$. This is clear, since the sequences $\left(M_{p}^{h}(\eta)\right)_{p=1}^{\infty}$ are increasing.

Corollary 6.2. Given any $J \in \mathbb{N}$, the spectrum (2.7) and the essential spectrum of (1.6)-(1.7) contain at least $J$ gaps, if $h$ is small enough.

Proof. Obviously, given any $J \in \mathbb{N}$, one can choose $J$ indices $j$ such that $\mu_{j}>\mu_{j-1}$ and then an $h>0$ small enough so that a gap is opened between $\Upsilon_{j-1}^{h}$ and $\Upsilon_{j}^{h}$ for all of these $j$, by the previous theorem.

\section{REFERENCES}

[1] Agmon, S., Douglis, A., Nirenberg, L., Estimates near the boundary for solutions of elliptic differential equations satisfying general boundary conditions 2, Comm. Pure Appl. Math. 17 (1964), 35-92.

[2] Alonso, A., Simon, B., The Birman-Krein-Vishik theory of selfadjoint extensions of semibounded operators, J. Operator Theory 4,2 (1980), 251-270.

[3] Bakharev, Nazarov, S.A., Ruotsalainen, K., On the spectrum of Neumann-Laplacian on a cylinder with periodically immersed obstacles. To appear.

[4] Birman, M.S, Solomyak, M.Z, Spectral theory of self-adjoint operators in Hilber space. Reidel Publ. Company, Dordrecht (1986).

[5] Cardone, G., Minutolo, V., and Nazarov, S.A., Gaps in the essential spectrum of periodic elastic waveguides, Zeitschrift für Angewandte Mathematik und Mechanik (ZAMM), in print. 
[6] Cardone, G., Nazarov, S.A., and Perugia, C., A gap in the continuous spectrum of a cylindrical waveguide with a periodic perturbation of the surface, submitted to Math. Nachr..

[7] Filonov N., Gaps in the spectrum of the Maxwell operator with periodic coefficients, Comm. Math. Physics. 240, 1-2(2003), 161-170.

[8] Friedlander, L., On the density of states of periodic media in the large coupling limit, Comm. Partial Diff. Equations 27 (2002), 355-380.

[9] Friedlander, L.; Solomyak, M. On the spectrum of narrow periodic waveguides, Russ. J. Math. Phys. 15,2 (2008), 238-242.

[10] Gelfand, I.M, Expansion in characteristic functions of an equation with periodic coefficients (in Russian), Dokl.Akad.Nauk SSSR 73 (1950), 1117-1120.

[11] Gohberg, I.M., Krein, M.G., Introduction to the theory of linear nonselfadjoint operators, Am. Math. Soc. Providence, RI (1969).

[12] Green, E.L., Spectral theory of Laplace-Beltrami operators with periodic metrics, J. Differential Equations 133 (1997), 15-29.

[13] Hempel R., Lineau K., Spectral properties of the periodic media in large coupling limit, Comm. Partial Diff. Equations 25 (2000), 1445-1470.

[14] Kato, T., Perturbation theory for linear operators, 2nd edition, Springer-Verlag, Berlin-New York (1976)

[15] Kondratiev V.A., Oleinik O.A., Boundary-value problems for the system of elasticity theory in unbounded domains. Korn's inequalities, Uspehi mat. nauk., 43 (1988), no. 5, 55-98. (English transl.: Russ. Math. Surveys 43 (1988), no.5, 65-119.)

[16] Kuchment, P., Floquet theory for partial differential equations (in Russian), Uspekhi Mat. Nauk 37,4, (1982), 3-52. (English transl. Russ. Math. Surveys 37, 4 (1982), 1-60.)

[17] Kuchment, P., Floquet theory for partial differential equations. Basel: Birchäuser, 1993.

[18] Figotin, A., Kuchment, P., Band-gap structure of spectra of periodic dielectric and acoustic media. I. Scalar model, SIAM J. Appl. Math. 56 (1996), 68-88. II. Two-dimensional photonic crystals, ibid. 56 (1996), 1561-1620.

[19] Ladyzhenskaya, O.A., Boundary value problems of mathematical physics. Springer Verlag , New York (1985).

[20] Lekhnitskii, S.G., Elasticity of an anisotropic body (in Russian), Nauka, Moscow (1977).

[21] Lions, J.L., Magenes, E., Non-homogeneus boundary value problems and applications (in french), Dunod, Paris (1968). (English transl.: Springe-Verlag, Berlin-Heidelberg-New York (1972).)

[22] Nazarov, S.A., Asymptotic theory of thin plates and rods. Reduction of dimension and integral estimates (in Russian), Nauchnaya Kniga, Novosibirsk (2002).

[23] Nazarov, S.A., Properties of spectra of boundary value problems in cylindrical and quasicylindrical domains, Sobolev Spaces in Mathematics, vol. II (Maz'ya V., Ed.) International Mathematical Series 9 (2008), 261-309.

[24] Nazarov, S.A., A gap in the continuous spectrum of an elastic waveguide, C. R. Mecanique 336 (2008), 751-756.

[25] Nazarov, S.A., Korn's inequalities for elastic junctions of massive bodies and thin plates and rods, Uspehi mat. nauk. 63,1 (2008), 143-217. (English transl.: Russ. Math. Surveys. 63,1 (2008), 109-153.)

[26] Nazarov S.A., The Rayleigh waves in an elastic half-layer with partly jammed periodic boundary, Dokl. Ross. Akad. Nauk. 423, 1 (2008), 56-61 (English transl.: Doklady Physics, 53, 11 (2008), 600-604.)

[27] Nazarov, S.A., Gap detection in the spectrum of an elastic periodic waveguide with a free surface, Zh. Vychisl. Mat. i Mat. Fiz. 49,2 (2009), 323-333. (English transl.: Comput. Math. and Math. Physics. 49,2 (2009) 332-343.)

[28] Nazarov, S.A., Opening a gap in the essential spectrum of the elasticity problem in a periodic half-layer, Algebra i analiz 21,2 (2009), 166-202. (English transl.: St. Petersburg Math. J. $21,2(2009)$.) 
[29] Nazarov, S.A., Asymptotics of solutions to the spectral Steklov problem in a domain with a blunted peak, Mat. Zametki. 86,4 (2009) (English transl.: Math. Notes. 84, 3-4 (2009).)

[30] Nazarov, S.A, Plamenevskii, B.A, Elliptic problems in domains with piecewise smooth boundaries, Walter be Gruyter, Berlin, New York (1994).

[31] Zhikov, V., Gaps in the spectrum of some elliptic operators in divergent form with periodic coefficients, Algebra i Analiz 16,5 (2004), 34-58. (English transl.: St. Petersburg Math. J. 16,5 (2005), 773-790.)

Institute of Mechanical Engineering Problems, V.O., Bolshoi PR., 61, 199178, St. Petersburg, Russia.

University of Oulu, Department of Electrical and Information Engineering, Mathematics Division, P.O. Box 4500, FI-90401 Oulu, Finland

University of Helsinki, Department of Mathematics and Statistics, P.O. Box 68, FI-00014 Helsinki, Finland. 